\title{
BMJ Open Scoping review protocol of service delivery-related interventions to improve maternal and newborn health in low-income and middle-income countries
}

\author{
Solange Mianda (D) , ${ }^{1}$ Olamide O Todowede (D) , ${ }^{2}$ Helen Schneider ${ }^{1,3}$
}

To cite: Mianda S,

Todowede 00, Schneider $\mathrm{H}$. Scoping review protocol of service delivery-related interventions to improve maternal and newborn health in low-income and middle-income countries. BMJ Open 2021;11:e042952. doi:10.1136/ bmjopen-2020-042952

- Prepublication history and additional material for this paper are available online. To view these files, please visit the journal online (http://dx.doi. org/10.1136/bmjopen-2020042952).

Received 21 July 2020 Revised 15 March 2021 Accepted 16 March 2021

D) Check for updates

(c) Author(s) (or their employer(s)) 2021. Re-use permitted under CC BY-NC. No commercial re-use. See rights and permissions. Published by BMJ.

${ }^{1}$ School of Public, University of the Western Cape, Cape Town, South Africa

${ }^{2}$ Department of Health Science Mental Health and Addiction Research Group (MHARG), University of York, York, UK

${ }^{3}$ South African Medical research Council (SAMRC) Service to System Extramural research Unit, Cape Town, South Africa

Correspondence to

Dr Solange Mianda;

smianda@uwc.ac.za

\section{ABSTRACT}

Introduction This review will explore the characteristics of service delivery-related interventions to improve maternal and newborn health in low-income and middle-income countries, comparing three common framing approaches of these interventions (referred to as archetypes), namely, quality improvement (QI), health system strengthening (HSS) and implementation science (IS), over the last 20 years.

Methods and analysis This study will review the literature on health service interventions from 2000 to 2020. This will be achieved by searching for English peerreviewed articles in the following electronic databases EBSCOhost, PubMed, Web of Science, MASCOT/Wotro Map of Maternal Health Research and Google scholar. We will develop a systematic search strategy using a combination of keywords and Boolean operators AND/OR. Eligibility screening and data extraction will be conducted by two independent reviewers, and disagreements resolved by a third independent reviewer. Analyses will be conducted in two steps, a quantitative and a qualitative phase. The quantitative phase will provide a descriptive profile of papers, including the category (QI, HSS, IS, mixed or other) of papers. In the follow-up qualitative phase, all three reviewers will independently code for key concepts in a subset of papers, jointly selected as representing each archetype, and analysed in batches until concept saturation is achieved.

Ethics and dissemination This review does not require ethical approval. The results will be published as an article in a peer-reviewed journal and presented to stakeholders involved in implementing health system interventions in maternal and newborn.

\section{INTRODUCTION}

Maternal and newborn mortality remains an important public health issue around the world. ${ }^{1}$ The commitment to achieving the millennium development goals (MDGs) in many countries, substantially improved access to maternal, newborn health services, reaching poor and vulnerable populations especially in low-income and middle-income
Strengths and limitations of this study

- Findings from this review will add to the body of evidence regarding the characteristics of quality improvement, health system strengthening and implementation science interventions, and the possibilities for bringing the fields together to take advantage of their complementary ideas and approaches for improving maternal and newborn health.

- To the best of our knowledge, this study will be the first to comparatively review the different approaches to service delivery-related interventions to improve maternal and newborn health.

- The findings are expected to be useful to national and international stakeholders for designing and implementing service delivery interventions to improve maternal and newborn health.

- The use of the Levac, Heather and O'Brien framework to guide the scoping review will ensure a clear methodological and transparent process that can be replicated.

- A major limitation will be the inclusion of only English language studies, and publication bias as a result of excluding grey literature.

countries (LMICs; countries with gross national income per capita calculated using the World Bank Atlas method between $\$ 1035$ or less and $\$ 12535$ in 2019). ${ }^{2} 3$ However, despite investments to improve maternal and newborn health, reducing maternal and newborn mortality remains a major challenge in many LMICs. ${ }^{4}$ The sustainable development goals seek to expand the gains of reduced maternal and child mortality, achieved during the MDG era by $2030,{ }^{1}$ through better quality of care coupled with expansion of health service coverage under the banner of universal health coverage. ${ }^{5}$

Diverse forms of health system interventions and approaches are being implemented in LMICs towards improved maternal and 
newborn care. ${ }^{6-8}$ These interventions are also becoming more complex, evolving from linear chains of causeand-effect, towards engaging interconnected elements holistically within complex systems and involving multistakeholder approaches. ${ }^{9} 10$ Evidence has shown the mutual benefits of involving a diversity of stakeholders such as policy makers, healthcare providers, researchers and funding organisations in implementing and/or evaluating complex health system interventions. ${ }^{11} 12$ There are three common service delivery-related approaches for improving maternal and newborn health: quality improvement (QI), health system strengthening (HSS) and implementation science (IS). ${ }^{513}$

The three approaches have different origins: QI applies pragmatic approaches from industry to reducing poor performance in health; HSS emerged from the field of global health concerned with the wider health system constraints to implement disease-specific or programmatic interventions ${ }^{14}$; and IS draws from behavioural science to focus on the adoption of evidence-based practice in healthcare. ${ }^{1315}$ Moreover, these approaches also use different designs, methodologies, concepts, terminologies, discourses, theories and frameworks. They operate with distinct professional societies, journals of publication, funding and training streams. They also focus on different levels: QI tends to be health facility based (micro-level), HSS operates at meso-level and macro-level (district, regional or national settings); while IS tends to be more focused on provider's behaviour while also typically referencing research methodologies such as cluster randomised trials. ${ }^{13}$ However, they share similar goals, namely, a systematic approach to behaviour change in order to improve healthcare practice and service delivery. ${ }^{1316}$ As such, each approach, QI, HSS or IS may offer ideas, concepts and methodologies that combined could benefit the field greatly. However, their similarities or relatedness are often not appreciated nor understood, and consequently, opportunities to leverage their combined strengths are missed. ${ }^{17}$

\section{STUDY OBJECTIVES}

This scoping review explores the scope of existing literature on QI, HSS and IS interventions to improve maternal and newborn health in LMICs, to draw out key concepts associated with each approach in order to appreciate their relatedness and distinctiveness, and establish underlying mechanisms associated with change.

\section{STUDY CONCEPT DEFINITION}

$Q I$ is defined as the combination of the efforts of multiple actors to make the changes required to lead to better outcomes, better system performance and professional development. ${ }^{18}$

HSS refers to the process of identifying and implementing changes in policy and practices in its health system, to respond better to its health and health system challenges. ${ }^{14}$

$I S$ is the scientific study of methods to promote translating systematic research findings into evidence-based practices, routine practice and policy development, to improve the quality and effectiveness of health services and care. ${ }^{19}$

\section{PROTOCOL DESIGN}

We will conduct this scoping review using the methodology proposed by Levac et $a l^{20}{ }^{20}$ to map literature on maternal and newborn interventions, with keywords referencing terminology associated with the QI, HSS and IS approaches. Levac et als framework builds on Arksey and O'Malley methodological framework ${ }^{21}$ with recommendations for clarification to enhance the methodology of scoping review studies. The use of this framework to guide the review will ensure clear methodological and transparent processes that can be replicated. The Preferred Reporting Item for Systematic Reviews and Meta-Analyses (PRISMA) extension for Scoping Reviews check list ${ }^{22}$ will be used throughout the review process, to guide the screening and reporting (see online supplemental file).

\section{Stage 1: Identifying the research question}

The concept, target population and outcomes framework will be used to formulate the review question as shown in table $1 .^{21}$

The overarching research question that will guide this scoping review is: What are the characteristics of QI, HSS or IS interventions used to improve maternal and newborn health in LMICs?

The subquestions are

1. What is the distribution of approaches (QI, HSS, IS, other or mixed) in the literature on maternal and newborn service delivery interventions?

Table 1 Concept, target group and outcomes framework to guide the research question

C-Concept

Quality improvement

Implementation science or

implementation research

Health system strengthening

Interventions

Model or framework

Constructs and concepts

Assumptions or theories

\begin{tabular}{ll} 
T-Target group & $\begin{array}{l}\text { Actors targeted for change } \\
\text { Stakeholders involved in change } \\
\text { Low-income and middle-income } \\
\text { countries }\end{array}$ \\
$\begin{array}{l}\text { Outcome (focus of } \\
\text { papers) }\end{array}$ & $\begin{array}{l}\text { Maternal health } \\
\text { Newborn health (encompassing the } \\
\text { range of processes and outcomes of } \\
\text { service delivery-related interventions) }\end{array}$ \\
\hline
\end{tabular}


2. Who are the actors targeted for change in QI, HSS or IS interventions to improve maternal and newborn health in LMICs?

3. Who are the (other) health system stakeholders involved in the change processes during QI, HSS or IS interventions to improve maternal and newborn health in LMICs?

4. What are the services or systems areas of focus of QI, HSS or IS interventions to improve maternal and newborn health in LMICs?

5. What are the key constructs and concepts used in QI, HSS or IS interventions to improve maternal and newborn health in LMICs?

6. What are the frameworks or models used in QI, HSS or IS interventions to improve maternal and newborn health in LMICs?

7. What are the theories or assumptions about change in QI, HSS or IS interventions to improve maternal and newborn health in LMICs?

\section{Stage 2: Identifying relevant studies—search strategy}

This stage will involve an iterative process involving searching the literature, refining the search strategy and reviewing articles for study inclusion. The search strategy was collaboratively developed by the review team, and the relevance of searched studies will be determined and agreed by all three researchers. The inclusion and exclusion criteria were refined and agreed by all three researchers. The literature search will include only peerreviewed articles published in English language.

The identification of literature will involve searching the electronic databases, using structured search terms, as applicable to the individual databases. The following electronic databases will be searched EBSCOhost, PubMed, Web of Science, MASCOT/Wotro Map of Maternal Health Research and Google scholar advance search.

We will develop a systematic search strategy using a combination of keywords and Boolean operators AND/ OR. We will limit our search to publication published between 2000 and 2020, to capture the growth of interest in the different approaches (QI, HSS and IS) that evolved in the era of the MDGs.

The search will be documented detailing the date, database, keywords and the number of articles retrieved. The search strategy will be piloted to check its suitability to selected databases and keyword. A PubMed pilot sample search is shown in online supplemental table 1 .

\section{Stage 3: Selection of relevant articles}

The review will use the concept, target population and outcomes framework to align the study selection with the research question. Further eligibility criteria will ensure that selected articles are relevant to the research question. Titles, abstract and full texts screening will be independently screened by two reviewers (SM and OOT) using screening tools designed by the review team. We will use EndNote to store, organise and manage the papers, and a google form will be developed and piloted for the screening and extraction of data at each screening stage. The screening stages will be continuously monitored guided by the inclusion and exclusion criteria described below. Double screening of titles/abstracts and full texts will be managed by calculating disagreement rates between the screeners. A third reviewer (HS) will be approached in case of disagreement on included articles, to resolve the matter and decide on the final inclusion. From this universe of papers, the three authors will jointly select 15 (5 each per category of QI, HSS and IS) as representing each archetype using a systematic sampling for further qualitative analysis. An additional five papers in the 'other' category (selected for concept diversity) will also be identified.

\section{Inclusion criteria}

The following criteria will guide the selection of study that will be included in the review:

- Articles exploring QI, HSS or IS interventions to improve maternal and newborn health.

- Studies published in English

- Studies published between 2000 and 2020

- Studies conducted within LMICs

\section{Exclusion criteria}

The following criteria will be considered in excluding studies from the review:

- Studies exploring interventions on cost-effectiveness

- Studies published in other languages because of limited resources for translation by the review team.

- Studies published before 2000.

- Studies conducted in high-income countries

- Grey literature because of time and resource constraints.

The PRISMA flow diagram will be used in the study selection process and will be updated once the review is completed (figure 1).

\section{Stage 4: Charting the data}

We will follow these steps in charting the data:

Step 1: The research team will collectively develop a data charting form using google forms, to extract data on the following variables: author, year of publication, country where the study was conducted, journal where the study was published, field of research, approach used, goal/purpose of the study, actors targeted for change, health system stakeholders involved, health system setting of focus, key assumptions, key constructs and key components of the intervention (see online supplemental file data extraction form). The review team will pilot the data charting form to test out the form and identify any detail that needs to be addressed before proceeding with the main data extraction process. Data charting will be an iterative process in which the review team will continually extract data and update the data charting form.

Step 2: Data extraction will proceed by conducting an inventory of variables and grouping them until saturation 


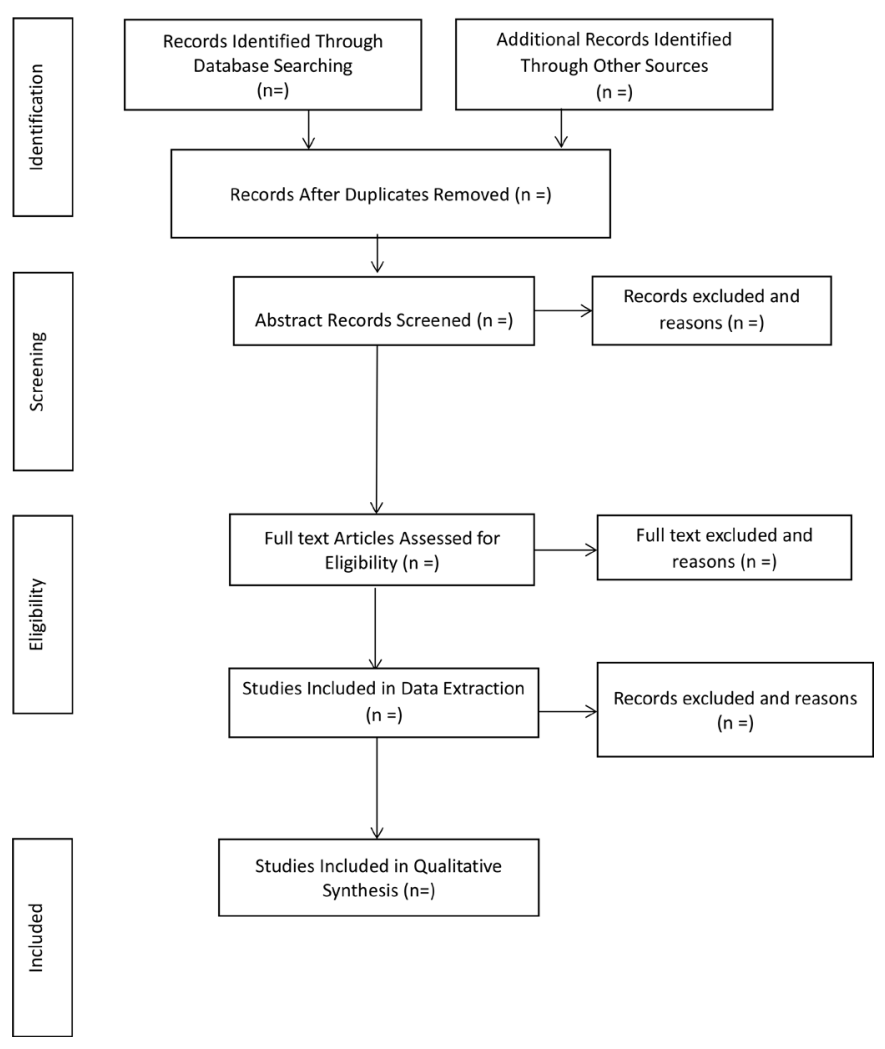

Figure 1 Preferred Reporting Items for Systematic Reviews and Meta-Analyses flowchart of study selection process.

is reached. Data will be analysed using quantitative and qualitative methods.

- Quantitative analysis will be performed (by SM and OOT) using word cloud to show the differences and range in variables based on the journal where the articles were published, countries and regions, field of research, approach, goal/purpose of the study, actors targeted for change, health system stakeholders involved and health system setting of focus.

- Qualitative analysis will involve all three reviewers in an in-depth review of a subset of studies, as outlined earlier. The research team will chart out the major concepts and processes that are used; afterwards, the concepts and processes will be compared across approaches.

\section{Stage 5: Collating, summarising and reporting of results}

A narrative report will be produced to summarise the data extracted around the following themes: similarities between the different approaches in relation to each variable, differences between the different approaches in relation to each variable, distinct characteristic of each approach and the underlying mechanism associated with change using each approach.

\section{Stage 6: Consultation}

This review is part of a project evaluating the implementation of a QI initiative to improve maternal and newborn health in three South African health districts. We intend to approach members of the project management team and some health district managers, on the outcome of the review to gain more insights on possible synergies between QI, HSS and IS and how these can be integrated into practice.

\section{ETHICS AND DISSEMINATION}

This review does not require ethical approval, as it will not involve access to individual level data. The results will be disseminated through peer-reviewed scientific journal, presentation to prospective maternal and newborn funders and practitioners to improve maternal and newborn outcomes in LMICs. We will report on the strength and limitations of our review and will provide recommendations and conclusions based on the findings from the synthesis.

\section{DISCUSSION}

This proposed scoping review aims to identify and describe the characteristics of QI, HSS and IS interventions to improve maternal and newborn outcomes in LMICs. To the best of our knowledge, this review will be the first to review the characteristics of QI, HSS and IS interventions and the possibilities for synergy between the three approaches to improve maternal and newborn outcomes. An understanding of the similarities and differences between these approaches, as well as key distinct features to each approach and associated mechanisms of change, can assist stakeholders involved in implementing health system interventions in maternal and newborn to leverage their combined strengths. This review also has the potential to show areas of possible synergy between QI, HSS and IS to improve maternal and newborn outcomes.

Acknowledgements The authors would like to acknowledge funding from the Belgian Development Cooperation, through the Institute of Tropical Medicine Antwerp.

Contributors SM and O0T were responsible for designing the review, including the search strategies, and producing the initial draft of the review protocol. HS proposed the review and is the third reviewer. All authors provided significant editorial comments on the protocol drafts and read and approved the final manuscript.

Funding This work is based on the research supported by the South African Research Chairs Initiative of the Department of Science and Technology and National Research Foundation of South Africa (grant no. 98918).

Disclaimer Any opinion, finding and conclusion or recommendation expressed in this material is that of the authors and not the funders.

Competing interests None declared.

Patient consent for publication Not required.

Provenance and peer review Not commissioned; externally peer reviewed.

Supplemental material This content has been supplied by the author(s). It has not been vetted by BMJ Publishing Group Limited (BMJ) and may not have been peer-reviewed. Any opinions or recommendations discussed are solely those of the author(s) and are not endorsed by BMJ. BMJ disclaims all liability and responsibility arising from any reliance placed on the content. Where the content includes any translated material, BMJ does not warrant the accuracy and reliability of the translations (including but not limited to local regulations, clinical guidelines, terminology, drug names and drug dosages), and is not responsible for any error and/or omissions arising from translation and adaptation or otherwise. 
Open access This is an open access article distributed in accordance with the Creative Commons Attribution Non Commercial (CC BY-NC 4.0) license, which permits others to distribute, remix, adapt, build upon this work non-commercially, and license their derivative works on different terms, provided the original work is properly cited, appropriate credit is given, any changes made indicated, and the use is non-commercial. See: http://creativecommons.org/licenses/by-nc/4.0/.

\section{ORCID iDs}

Solange Mianda http://orcid.org/0000-0002-4353-3929

Olamide 0 Todowede http://orcid.org/0000-0002-5785-1426

\section{REFERENCES}

1 Zeng W, Li G, Ahn H, et al. Cost-Effectiveness of health systems strengthening interventions in improving maternal and child health in low- and middle-income countries: a systematic review. Health Policy Plan 2018;33:283-97.

2 Alkenbrack S, Chaitkin M, Zeng W, et al. Did equity of reproductive and maternal health service coverage increase during the mdg era? an analysis of trends and determinants across 74 low- and middleincome countries. PLoS One 2015;10:e0134905.

3 World Bank. Country income groups (world bank classification), country and lending groups, 2011. Available: http://data.worldbank. org/about/country-classifications/country-and-lending-groups

4 Kung'u JK, Ndiaye B, Ndedda C, et al. Design and implementation of a health systems strengthening approach to improve health and nutrition of pregnant women and newborns in Ethiopia, Kenya, niger, and Senegal. Matern Child Nutr 2018;14 Suppl 1:e12533.

5 Goyet S, Broch-Alvarez V, Becker C. Quality improvement in maternal and newborn healthcare: lessons from programmes supported by the German development organisation in Africa and Asia. BMJ Glob Health 2019;4:e001562-9.

6 Schneider H, George A, Mukinda F, et al. District governance and improved maternal, neonatal and child health in South Africa: pathways of change. Health Syst Reform 2020;6:e1669943.

7 Magge $\mathrm{H}$, Chilengi R, Jackson EF, et al. Tackling the hard problems: implementation experience and lessons learned in newborn health from the African health Initiative. BMC Health Serv Res 2017;17:39-52

8 Waiswa P, O'Connell T, Bagenda D, et al. Community and district Empowerment for scale-up (codes): a complex district-level management intervention to improve child survival in Uganda: study protocol for a randomized controlled trial. Trials 2016;17:135.

9 Moore GF, Audrey S, Barker M, et al. Process evaluation of complex interventions: medical Research Council guidance. BMJ 2015;350:h1258-7

10 Craig P, Dieppe P, Macintyre S, et al. Developing and evaluating complex interventions: the new medical Research Council guidance. BMJ 2008;337:a1655-983.

11 Bullock A, Morris ZS, Atwell C. Collaboration between health services managers and researchers: making a difference? J Health Serv Res Policy 2012;17 Suppl 2:2-10.

12 Hirschhorn LR, Baynes C, Sherr K, et al. Approaches to ensuring and improving quality in the context of health system strengthening: a cross-site analysis of the five African health Initiative partnership programs. BMC Health Serv Res 2013;13:1-11.

13 Koczwara B, Stover AM, Davies L, et al. Harnessing the synergy between improvement science and implementation science in cancer: a call to action. J Oncol Pract 2018;14:335-40.

14 Executive Board 128th. Health System Strengthening - Current Trends and Challenges. Geneva, 2011. http://apps.who.int/gb/ ebwha/pdf_files/EB128/B128_37-en.pdf

15 Davidoff F, Dixon-Woods M, Leviton L, et al. Demystifying theory and its use in improvement. BMJ Qual Saf 2015;24:228-38.

16 Witter S, Palmer N, Balabanova D, et al. Health system strengthening-Reflections on its meaning, assessment, and our state of knowledge. Int J Health Plann Manage 2019;34:e1980-9.

17 Moore J. The missing link: how implementation science and quality improvement can team up to improve care. implementation in action bulletin, 2019. Available: https://quorum.hqontario.ca/en/Home/ Posts/The-missing-link-How-can-implementation-science-andquality-improvement-team-up-to-improve-care-1 [Accessed $10 \mathrm{Nov}$ 2020].

18 Batalden PB, Davidoff F. What is "quality improvement" and how can it transform healthcare? Qual Saf Health Care 2007;16:2-3.

19 Eccles MP, Mittman BS. Welcome to implementation science. Implementation Sci 2006;1:1.

20 Levac D, Colquhoun H, O'Brien KK. Scoping studies: advancing the methodology. Implement Sci 2010;5:69.

21 Arksey H, O'Malley L. Scoping studies: towards a methodological framework. Int J Soc Res Methodol 2005;8:19-32.

22 Tricco AC, Lillie E, Zarin W, et al. PRISMA extension for scoping reviews (PRISMA-ScR): checklist and explanation. Ann Intern Med 2018;169:467-73. 